\title{
Antimicrobial Stewardship Program at a tertiary teaching hospital in Vietnam: A longitudinal observational study
}

\author{
Nguyen Truong Son ${ }^{1 *}$, Ton Thanh Tra ${ }^{2}$, and Pham Thi Ngoc Thao ${ }^{3}$ \\ ${ }^{1}$ Director of Cho Ray hospital, Ho Chi Minh City, Vietnam, President of Antimicrobial Stewardship Program at Cho Ray hospital, Vietnam \\ ${ }^{2}$ Head of quality management department, Cho Ray hospital, Ho Chi Minh City, Vietnam - Coordinator of Antimicrobial stewardship program at Cho Ray \\ hospital, Vietnam \\ ${ }^{3}$ Vice - director, Cho Ray hospital, Ho Chi Minh City - Vietnam. Vice president of Antimicrobial Stewardship Program at Cho Ray hospital, Vietnam
}

\begin{abstract}
Introduction: Antimicrobial resistance is an emerging global problem, particularly in developing countries. Cho Ray is one of the first hospitals in Vietnam to implement an antimicrobial stewardship program. We present the results of this program here, after two years of implementation.

Objectives: To determine the compliance rate of hospital antibiotic guidelines, the cost savings of antibiotic usage and the incidence of hospital acquired infection.

Materials and methods: A prospective, longitudinal study was done from January 2016 through December 2016. All inpatients prescribed antibiotics during their treatment were enrolled.

Results: 2,472 medical records were audited, of which 1,664 comprised instances of antibiotic treatment, and 808 comprised instances of antibiotic prophylaxis for surgery. The compliance rate to the hospital antibiotic guideline was $77.5 \%$, an increase of $14.5 \%$ compared with 2015 ( $<<0.05)$. The overall treatment response rate was $87.7 \%$. The antibiotic cost was $17.2 \%$ of the total pharmaceutical budget, reduced by $1.3 \%$ compared with 2015 ( $\mathrm{p}<0.05$ ). The hospital infection incidence was $2.9 \%$, and the hospital infection rate per 1,000 patient-days was $1.8 \%$, while the surgical site infection rate was $4.0 \%$.

Conclusions: The Antibiotic Stewardship Program at Cho Ray hospital has shown initially promising results. The hospital antibiotic compliance rate increased by $14.5 \%$, and the antibiotic cost was reduced by $1.3 \%$, compared with 2015 . The treatment response rate remained the same, and rates of hospital infection did not increase, compared with previous years.
\end{abstract}

\section{Introduction}

Bacterial resistance is an emerging global health problem. Resistant bacteria have been responsible for approximately 50,000 deaths annually during the past decade in Europe and the United States of America [1]. This number will increase to as many as 10 million as a result of antimicrobial resistance, by the year 2050 , by some estimates [1]. Antibiotic stewardship programs (hereinafter, ASP) are designed to optimize antimicrobial usage to improve patient care, limit antimicrobial resistance, and reduce overall healthcare costs [2,3]. ASP has been shown to reduce antimicrobial consumption by as much as $36 \%$, and to provide annual cost reductions of up to 900,000 USD, in the past [4]. Extensive research in developed countries has shown that antibiotic agents comprise up to $30 \%$ of total medication costs in many institutions [5]. However, up to $30 \%$ of this antibiotic usage is inappropriate, owing to the development of bacterial resistance [6].

Vietnam is a country with a high prevalence of bacterial resistance. In 2016 the Vietnam Ministry of Health instituted a national program aimed at preventing the spread of resistance including an antibiotic awareness week, antibiotic national guidance committee, and the drafting of best practice antimicrobial guidelines. Uptake, however, has been slow. Located in the South of Vietnam, Cho Ray is a public tertiary teaching hospital containing 2,600 beds. In 2015, the bed capacity was $98 \%$, with additional services for $1,200.000$ outpatients per year. The mean length of stay for all admissions was 7.0 days in 2016. Cho Ray hospital has 38 clinical departments and four central laboratories. About $50 \%$ of inpatients are referred from provincial hospitals. An ASP was originally implemented in January 2015 with six pilot departments, and then increased to all clinical departments in 2016. The aim of this manuscript was to determine the compliance rate of hospital antibiotic guidelines, the cost savings of antibiotic usage, and the incidence of hospital acquired infection.

\section{Materials and methods}

Data were collected from January 2016 through December 2016, in all departments at Cho Ray hospital. All inpatients that were given antibiotics for treatment, or as prophylaxis for surgery, were enrolled. Departments, and individual medical records within each department, were randomized by hospital number. Patient stratification, surgical site classification, hospital antibiotic guideline compliance, clinical response, and routine microbiology were all recorded. The defined daily dose of antibiotics and total antibiotic cost were collected from the pharmacy department. The antibiotic drug prices provided reflect

Correspondence to: Nguyen Truong Son, Director of Cho Ray hospital, Ho Chi Minh City, Vietnam, President of Antimicrobial Stewardship Program at Cho Ray hospital, Vietnam, Tel: +84989108268; E-mail: truongson_cr@yahoo.com.vn

Key words: antimicrobial stewardship program, compliance, cost, vietnam

Received: January 17, 2017; Accepted: February 23, 2017; Published: February 27,2017 
the actual prices charged by pharmaceutical companies in Vietnam. The hospital infection data were collected from the infection control department in all clinical departments. Treatment responses were based on clinical presentations, laboratory tests, imaging tests, and microbiology. SPSS 16.0 was used to analyze the results. Significance was defined by $\mathrm{p}<0.05$ using the $\mathrm{T}$ test.

\section{Ethics}

Our project was prior-approved by the Cho Ray ethics and scientific committee (N 100/CRH, dated 20/11/2015).

\section{Results}

2,472 medical records were audited, of which 1,664 comprised instances of antibiotic treatment, and 808 comprised instances of antibiotic prophylaxis for surgery.

Table 1 shows clinical distribution of the number of patients and the percentage of total, based on treating department. Most patients were in internal medicine (46.0\%) and surgical departments (40.8\%).

Table 2 shows the recorded origin of infection for inpatients in all departments showing health care associated infections $(43.0 \%)$, hospital acquired infections (30.7\%) and community acquired (26.3\%),

\section{Antibiotic prophylaxis for surgery}

The program covered all surgical departments. Patients who underwent a clean operation or clean-dirty operation were selected. Dirty operations (because of trauma) were excluded, owing to nondiscretionary antibiotic use.

Table 3 shows the number of patients and percent of total cohort by surgical classification. The antibiotic prophylaxis applied to clean operations and clean - dirty operations. In our study, 93\% patients were classified, $60.5 \%$ was clean operation.

Figure 1 shows monthly guideline compliance (percentage) of all departments. The mean value was $77.5 \%$. The first 3 months of audit, the compliance rate was $56.0-66.0 \%$ but the last 3 months of year, the compliance rate was 81.0 - $86.3 \%$.

Table 4 shows hospital antibiotic guidelines compliance as a percentage of all cases by year. The compliance rate to the hospital antibiotic guidelines was $77.5 \%$, an increase of $14.5 \%$ compared with $2015(\mathrm{p}<0.05)$ and $48.6 \%$ in antibiotic prophylaxis compared with 2015 $(\mathrm{p}<0.05)$

Table 5 shows treatment results by number of patients, and as percentage of total inpatients. The response to treatment for empiric antibiotic therapy according to hospital antibiotic guideline was $87.7 \%$ (good response and reduced infection). The response of treatment was the same previous years $(\mathrm{p}<0.05)$

Table 6 shows the rate of patients requiring antibiotic treatment and combined antibiotic drug cost saving comparing with the previous year across all departments by year. The antibiotic cost was $17.2 \%$ of the total pharmaceutical budget in 2016, reduced by $1.3 \%$ compared with $2015(\mathrm{p}<0.05)$. The cost saving was 2.1 million USD in 2015 and additional 1 million USD in 2016.

\section{Bacterial resistance}

Table 7 shows the common identified antibiotic resistant bacteria across all departments as a percentage of total identifications, by month, over the course of 2016. Acinetobacter Baumanii was the most antibiotic resistant bacteria identified in 2016.
Table 1. Clinical distribution of the number of patients based on treating department.

\begin{tabular}{|c|c|c|}
\hline Clinical departments & Number & $\mathbf{( \% )}$ \\
\hline Internal medicine departments & 766 & $(46.0)$ \\
\hline Surgical medicine departments & 679 & $(40.8)$ \\
\hline Intensive care units & 142 & $(8.6)$ \\
\hline High quality service departments & 77 & $(4.6)$ \\
\hline Total & 1,664 & $(100)$ \\
\hline
\end{tabular}

Table 2.Recorded origin of infection for inpatients in all departments.

\begin{tabular}{|c|c|c|}
\hline Type of infection & Number & $\mathbf{( \% )}$ \\
\hline Healthcare associated infection & 676 & $(43.0)$ \\
\hline Hospital acquired infection & 483 & $(30.7)$ \\
\hline Community acquired infection & 413 & $(26.3)$ \\
\hline Total & 1,572 & $(100)$ \\
\hline
\end{tabular}

Table 3.Number of patients and percent of total cohort by surgical classification.

\begin{tabular}{|c|c|c|}
\hline Type of operation & Number & $\mathbf{( \% )}$ \\
\hline Clean operation & 489 & $(60.5)$ \\
\hline Clean-dirty operation & 262 & $(32.5)$ \\
\hline Non-classification & 57 & $(7.0)$ \\
\hline Total & 808 & $(100)$ \\
\hline
\end{tabular}

Table 4.Hospital antibiotic guideline compliance as a percentage of all cases by year.

\begin{tabular}{|c|c|c|c|}
\hline \multirow{2}{*}{ Type of treatment } & \multicolumn{2}{|c|}{$\begin{array}{c}\text { Hospital antibiotic guideline } \\
\text { compliance (\%) }\end{array}$} & \multirow{2}{*}{ p } \\
\cline { 2 - 3 } & $\mathbf{2 0 1 5}$ & $\mathbf{2 0 1 6}$ & \\
\hline Treatment antibiotic & 63.0 & 77.5 & $<0.05$ \\
\hline Prophylaxis antibiotic & 17.0 & 65.6 & $<0.05$ \\
\hline
\end{tabular}

Table 5.Treatment results by number of patients, and as percentage of total inpatients.

\begin{tabular}{|c|c|c|}
\hline Result of treatment & Number & $\mathbf{( \% )}$ \\
\hline Good response & 983 & $(59.1)$ \\
\hline Reduced infection & 476 & $(28.6)$ \\
\hline No response & 205 & $(12.3)$ \\
\hline Total & 1664 & $(100)$ \\
\hline
\end{tabular}

Table 6. Rate of patients requiring antibiotic treatment and combined antibiotic drug cost saving comparing with the previous year across all departments by year.

\begin{tabular}{|c|c|c|c|c|c|}
\hline Year & $\mathbf{2 0 1 3}$ & $\mathbf{2 0 1 4}$ & $\mathbf{2 0 1 5}$ & $\mathbf{2 0 1 6}$ & $\mathbf{p}$ \\
\hline $\begin{array}{c}\text { Antibiotic use } \\
\text { rate }\end{array}$ & $21.3 \%$ & $20.4 \%$ & $18.5 \%$ & $17.2 \%$ & $<0.05$ \\
\hline Cost saving & n.a. & n.a. & 2.1 million USD & 1.0 million USD & \\
\hline
\end{tabular}

n.a.: not applicable

\section{Hospital infection}

Figure 2 shows the hospital infection rates divided by total admissions (blue), hospital infection rates divided by 1,000 patientsdays, and trend-line $\left(\mathrm{R}^{2}=0.0077\right)$. The hospital infection incidence was $2.9 \%$, and the hospital infection rate per 1,000 patient-days was $1.8 \%$. There was no significant difference in hospital infection rate between 2015 and 2016 ( $>>0.05)$.

Table 8 shows the commonly prescribed antibiotic drugs in 2016 by defined daily dose and as percentage of total antimicrobial prescriptions. The most 9 antibiotic drugs by defined daily dose were the new generation antibiotics.

\section{Discussion}

Our study demonstrates that a rational prescription framework based on stratifying surgical patients by risk for surgical site infection, and by promoting the appropriate use and de-escalation of targeted therapies, for medical inpatients, can increase the public guideline 


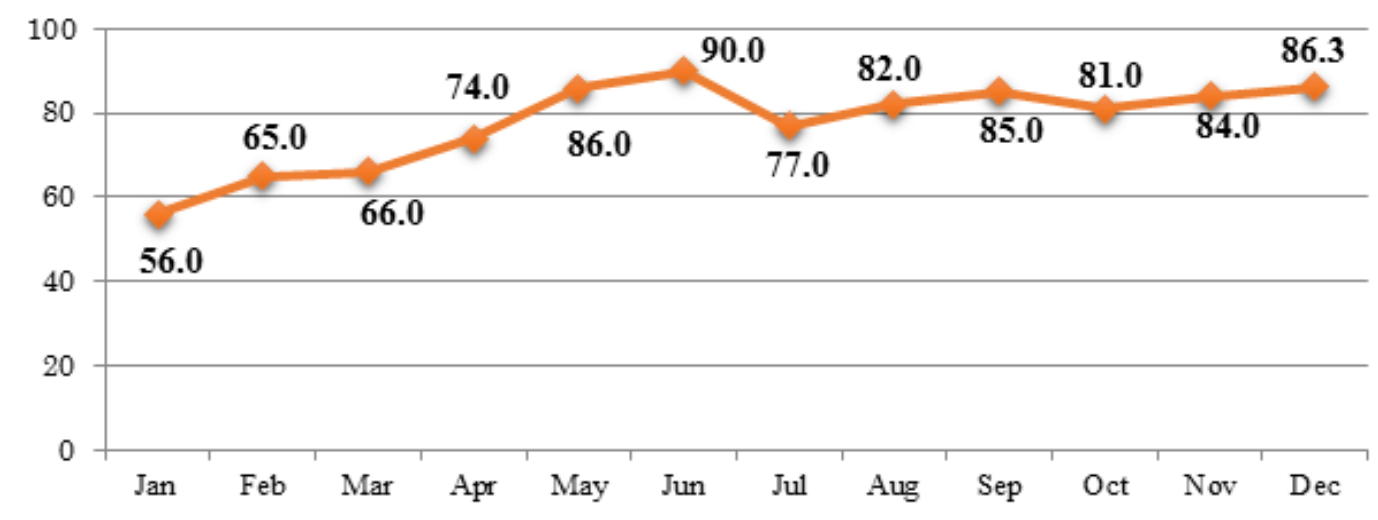

Figure 1. Monthly guideline compliance (percentage) of all departments in 2016 (mean $77.5 \%$ ).

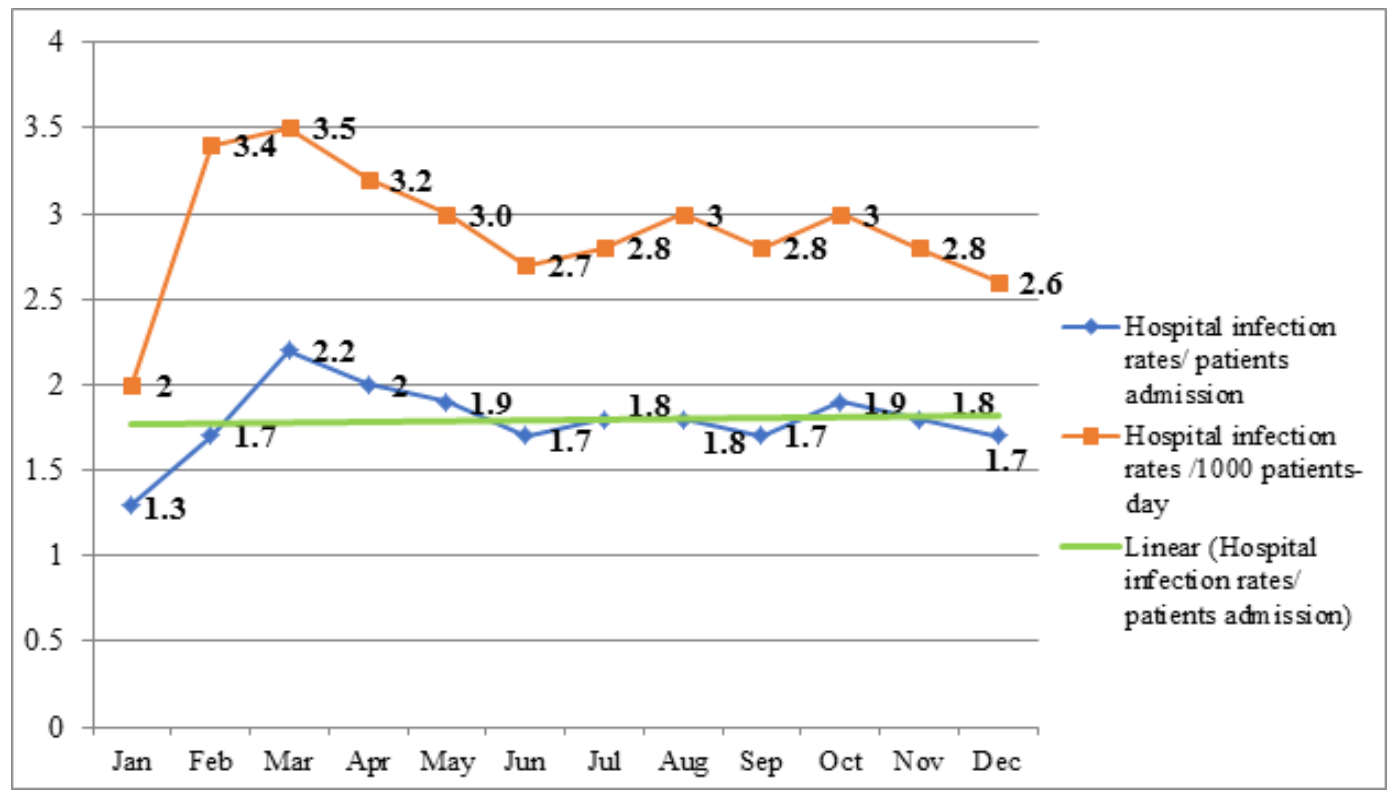

Figure 2. Hospital infection rates divided by total admissions (blue), hospital infection rates divided by 1,000 patient-days in 2016, and trend-line $\left(\mathrm{R}^{2}=0.0077\right)$.

Table 7. Common identified antibiotic resistant bacteria across all departments as a percentage of total identifications, by month, over the course of 2016.

\begin{tabular}{|c|c|c|c|c|c|c|c|c|c|c|c|c|c|}
\hline & Jan & Feb & Mar & Apr & May & Jun & Jul & Aug & Sep & Oct & Nov & Dec & Mean \\
\hline A. baumannii & 37.5 & 43.6 & 32.9 & 28.7 & 33.9 & 29.2 & 30.2 & 32.4 & 31.9 & 31.4 & 29.8 & 32.0 & 32.7 \\
\hline E. coli & 25.0 & 14.5 & 23.0 & 22.4 & 25.1 & 21.4 & 24.7 & 23.0 & 20.5 & 24.6 & 21.3 & 21.7 & 22.3 \\
\hline S. aureus & 18.8 & 18.9 & 17.4 & 19.6 & 17.9 & 20.6 & 18.3 & 18.2 & 22.9 & 14.4 & 14.5 & 18.8 & 18.3 \\
\hline K. pneumoniae & 11.3 & 10.1 & 13.3 & 11.7 & 8.5 & 13.0 & 9.4 & 11.3 & 10.9 & 12.5 & 13.4 & 15.1 & 11.7 \\
\hline
\end{tabular}

Table 8.Commonly prescribed antibiotic drugs in 2016 by defined daily dose and as percentage of total antimicrobial prescriptions.

\begin{tabular}{|c|c|c|}
\hline Antibiotic drugs & Defined Daily Dose & $\mathbf{( \% )}$ \\
\hline Imipenem + Cilastatin & 152,479 & $(5.9)$ \\
\hline Teicoplanin & 85,160 & $(3.3)$ \\
\hline Amikacin & 56,695 & $(2.2)$ \\
\hline Meropenem & 49,466 & $(1.9)$ \\
\hline Vancomycin & 44,916 & $(1.8)$ \\
\hline Ertapenem & 26,583 & $(1.0)$ \\
\hline Colistin & 25,726 & $(1.0)$ \\
\hline Doripenem & 9453 & $(0.4)$ \\
\hline Ticarcilline+ Clavulanic & 3 & $(0.01)$ \\
\hline Others & 211,904 & $(82.5)$ \\
\hline
\end{tabular}

compliance rate, and reduce antibiotic cost, while preserving hospital infection rates to the same or a lower rate than previous years.

Patient characteristics: In our study, 30.7\% patients in our cohort were at a high risk for antimicrobial resistance, and were being treated for a hospital acquired or healthcare associated infection. Only $26.3 \%$ had community acquired infections (Table 2). As a tertiary hospital, we receive many of trauma patients who once admitted with wound infection or soiled wounds, require non-discretionary antibiotic treatment. In this study, we randomized clinical departments and individual patients that were treated with a course of antibiotic drugs. The number of patients audited depended on how long the patients stayed at the hospital, and how long each antibiotic agent was used. 
For antibiotic prophylaxis cases, we selected both clean and cleandirty operations. Most of these (89\%) were elective operations (data not shown). To implement the antibiotic stewardship program, we introduced the regulation that all surgeries must be classified by risk of surgical site infection, with a corresponding recommendation for appropriate antibiotic prophylaxis. Anesthetists controlled antibiotic usage during the post-operative period. Stewardship was implemented by providing antibiotic treatment only when clear evidence of infection was present.

Hospital antibiotic guideline compliance: We audited six pilot departments (three internal medicine, three surgical departments). These six covered the about $70-80 \%$ of infectious disease cases at Cho Ray hospital, in 2015. Sepsis, urinary tract infection, abdominal cavity infection, pneumonia, and soft tissue infection were the most common diseases. Compliance to new ASP recommendations showed an overall monthly increase. Compliance in the first 3 few months was $56-66 \%$, but steadily increased over the remaining 6 months of the year to $80 \%$ (Figure 1).

Antibiotic prophylaxis: We only audited treating antibiotics during the first nine months of 2015, while data on antibiotic prophylaxis were added in the remaining three months. Since October 2015, all elective operations had been given an ASP risk classification. During this period the Ministry of Health also issued national guidelines on antibiotic usage, which included antibiotic prophylaxis. Despite this, antibiotic prophylaxis compliance was very low (17\%).

Most surgeons gave post operation antibiotics without evidence of infection, which is a common issue in many hospitals in Vietnam due to the lack of standardized and consistently enforced best practice guidelines. In 2016 we audited all operative cases and showed the actual surgical site infection rate was only $4.0 \%$ when antibiotic prophylaxis compliance guidelines were followed in clean and cleandirty operations. At the end of 2016 the antibiotic prophylaxis rate in compliance with the newly implemented ASP was 65.6\%. Remaining cases were wrong doses, routes, or times of administration. To increase compliance the emerging use of information technology should be leveraged with integration to the hospital information system. This will provide immediate guidance to physicians, and flag inappropriate usage and dosing to clinical pharmacists. Electronic medical records represent an ideal vehicle for prospective audit and feedback. Ideally, coupled with this, the hospital antibiotic guidelines need be updated every three years, based on the local microbiology and antibiotic resistance situation.

Additionally, to increase compliance real-time consultation should be considered in future deployments of electronic infrastructure. Sick et al. [7] reported in 2013 that a preapproval antibiotic stewardship program at a tertiary care pediatric hospital in US saved 103,787 USD (95\% Confidence Interval: CI, $\$ 98,583-\$ 109,172$ ) per year, or 14,156 USD (95\% CI, $\$ 13,446-\$ 14,890)$ per 1,000 patient-days. However, any electronic infrastructure is only as good as underlying information system support. In our own institution, we retrospectively audit recorded instances of antibiotic use and report monthly. However, our work is constrained by a staff of only six part-time pharmacists available to review randomized medical records.

Hospital infection: During the study period the compliance of antibiotic use and antibiotic prophylaxis markedly increased. The overall antibiotic usage rate was reduced $1.3 \%$, without a corresponding increase in surgical site infections, staying constant at a rate of $4.0 \%$. The rate of hospital infection per admitted patient was $2.9 \%$, and the hospital infection per 1,000 patient-days was $1.8 \%$, while using reduced antibiotic prescription rates in line with the ASP. This is very promising, as antibiotic usage, and overall drug cost, were both considerably reduced during this time, without adverse effect on clinical outcomes.

Bacterial resistance: The most common bacteria isolated were A. Baumannii, E. coli, S. aureus, and K. pneumoniae. These represent the most common pathogens in tertiary hospitals in Vietnam. Experience shows the prevention of an increased burden of hospital acquired infection by these pathogens can be achieved economically through contact precaution, hand hygiene, use of personal protective equipment, and medical equipment disinfection, coupled with appropriate antibiotic usage to counter emerging resistance [8].

Antibiotic cost: The combined cost of all antibiotic agents was $17.2 \%$ of our pharmaceutical budget at our institution in 2016. With the implementation of restricted prescribing based on the ASP, this cost was reduced $1.9 \%$ relative to 2015 , with continuing reduction in 2016 of additional 1.3\%. This represents an equivalent cost saving of 2.1 million USD in 2015, and 1.0 million USD in 2016. Our experience correlates closely with other examples reported in the literature. Database surveys show that most tertiary hospitals spend about $30 \%$ of all medication costs on antibiotics [9]. Hersh et al. [10] conducted a study in eight pediatric hospitals across the USA in 2015 showing that the introduction of their antimicrobial stewardship guidelines realized cost reductions of $11 \%$ of the total pharmaceutical budget in 2007, up to $8 \%$ in $2012, p=0.04$. An economic analysis by Standiford et al. [11] in another USA hospital showed that ASP guidelines implemented in 2001 realized a three-year cost savings of 3 million USD, with cost increases of 2 million USD, in the two years after the ASP was rolled back. Additional research by Lanbeck et al. [12] showed that the first and second year of ASP use cost savings were USD 606,000 respectively. The necessity of rational prescribing guidelines for antimicrobials is reinforced when the cost of resistant infections is considered. Akpan et al. [13] reported that a single case of a drug-resistant Candida sp. fungus can result in 3-13 additional days of hospitalization, and incur a total of 6,000-29,000 USD in direct health care costs in the USA. Arif et al. [14] performed a meta-analysis in 2016, concluding that current data show the ASP to be a top priority in all hospitals in the world. Antimicrobial stewardship has been shown to not only offer cost effectiveness in acute hospitals, but also in long term care facilities [15]

ASP Model: The ASP Board in our hospital draws on the expertise of the director of the hospital, clinical microbiologists, infectious disease specialists, clinical pharmacists, and our head of pharmacy department, head of clinical departments, head of quality management, and head of infection control department. The ASP team meet monthly to review the overall antibiotic usage in all inpatients, and to discuss with junior physicians' appropriate antimicrobial strategy and deescalation therapy selection, and microbiology result interpretation [2]. We sought to apply the model of previously successful ASPs reported from developed countries, and then modified those to our local conditions [16]

We performed a pilot study in six clinical departments in 2015, and then expanded the study to all clinical departments in 2016. However, to maximize effectiveness we need information technology support, and a national database should be created such as Malaysian system [17].

The ASP model can be applied in tertiary hospitals, but also in lower level and community hospitals [18]. We have demonstrated that such a rational prescribing program for antimicrobials can be successfully implemented at the tertiary hospital level in Vietnam; however, our 
model can be transferred to lower level regional hospitals with minimal outlay. Although currently limited by a lack of information technology and electronic medical record services in Vietnam, future possibilities are promising.

Potential cost savings are considerable from the reduction of inappropriate prescriptions and resistant infection [19]. Our ASP, in its current form, which relies upon printed guidelines for rational prescribing and mandatory stratification of infection risk before procedures are performed, can be applied in other hospitals with limited resources. To implement this program effectively developing countries should complement individual institutional policies with strategy and information sharing at the national level incorporating the seven core elements of antimicrobial stewardship produced by the CDC for rational prescribing [20]. Two considerable challenges facing the introduction of ASP in developing countries are a lack of finance and insufficient information technology. However, our experience shows that with greater hospital commitment from physician to management level, team communication, involvement of clinical pharmacists, and coordination at the institutional level, these programs can still show significant results. From 2010 to 2014, despite access to local microbiology data, training courses, and antibiotic guidelines, inappropriate antibiotic usage was common. Since the 2015 implementation of our integrated ASP, coupled with monthly audits, compliance has increased sharply month by month.

Before the implementation of our integrated ASP, attitudes towards rational antimicrobial use in surgery remained stubbornly skeptical, despite ample evidence of overuse. To address this, future ASP programs should leverage administrative policies and prescription regulations, but also support vigilant local surveillance for emerging resistance. At the beginning of our study (2015), hospital antibiotic guideline compliance was $17.0 \%$, but at the end of the year 2016, the compliance rate was $65.6 \%$ (Table 4).

Cho Ray is the first hospital in Vietnam to implement a comprehensive ASP. We hope that the result of lowered overall antibiotics prescriptions and lessened costs, without a corresponding decline in patient outcome, will encourage other hospitals to implement an ASP. The benefit from the program will be maximized if all other hospitals in the region implement their own ASP to tackle emerging resistance at the regional level [21].

\section{Conclusions}

Our implementation of an ASP at a tertiary hospital in Vietnam has had good initial results. The compliance rate increased over the two-year study cycle (an increase of $14.5 \%$ for treatment antibiotic and $48.6 \%$ prophylaxis antibiotic). Antibiotic costs were reduced $(1.3 \%$ compared with 2015), and the hospital infection rate remained the same as during previous years. An ASP should be implemented continuously at other hospitals in Vietnam and other developing countries.

\section{Author contributions}

Each author contributed equally to the design, development, writing, and editing of the manuscript.

\section{Acknowledgements}

The authors appreciate Dr Koji Wada (National Center for Global Health and Medicine, Japan) for technical input for this manuscript.

\section{References}

1. Oberjé E, Tanke M, Jeurissen P (2016) Cost-Effectiveness of Policies to Limit Antimicrobial Resistance in Dutch Healthcare Organisations. Celsus Academie voor Beta Albare Zorg Pp: 7-8.
2. Dik JW, Hendrix R, Friedrich AW, Luttjeboer J, Panday PN, et al. (2015) CostMinimization Model of a Multidisciplinary Antibiotic Stewardship Team Based on a Successful Implementation on a Urology Ward of an Academic Hospital. PLoS One 10: $\mathrm{e} 0126106$.

3. Wenzler E, Wong JR, Goff DA, Jankowski CA, Bauer KA (2016) Controversies in Antimicrobial Stewardship: Focus on New Rapid Diagnostic Technologies and Antimicrobials. Antibiotics 5: E6.

4. Wong JR, Bauer KA, Mangino JE, Goff DA (2012) Antimicrobial Stewardship Pharmacist Interventions for CoagulaseNegative Staphylococci Positive Blood Cultures Using Rapid Polymerase Chain Reaction. Ann Pharmacother 46: 1484-1490. [Crossref]

5. Leuthner KD, Doern GV (2013) Antimicrobial stewardship programs. J Clin Microbiol 51: 3916-3920. [Crossref]

6. Nowak MA, Nelson RE, Breidenbach JL, Thompson PA, Carson PJ (2012) Clinical and economic outcomes of a prospective antimicrobial stewardship program. Am J Health-Syst Pharm 69: 1500-1508. [Crossref]

7. Sick AC, Lehmann CU, Tamma PD, Lee CK, Agwu AL (2013) Sustained savings from a longitudinal cost analysis of an internet-based preapproval antimicrobial stewardship program. Infect Control Hosp Epidemiol 34: 573-580. [Crossref]

8. Chen CH, Lin LC, Chang YJ, Chen YM, Chang CY, et al. (2015) Infection Control Programs and Antibiotic Control Programs to Limit Transmission of Multi-Drug Resistant Acinetobacter baumanniiInfections: Evolution of Old Problems and New Challenges for Institutes. Int J Environ Res Public Health 12: 8871-8882.

9. Walia K, Ohri VC, Mathai D; Antimicrobial Stewardship Programme of ICMR (2015) Antimicrobial stewardship programme (AMSP) practices in India. Indian J Med Res 142: 130-138. [Crossref]

10. Hersh AL, De Lurgio SA, Thurm C, Lee BR, Weissman SJ, et al. (2015) Antimicrobia Stewardship Programs in Freestanding Children's Hospitals. Pediatrics 135: 33 -39. [Crossref]

11. Standiford HC, Chan S, Tripoli M, Weekes E, Forrest GN (2012) Antimicrobial Stewardship at a Large Tertiary Care Academic Medical Center: Cost Analysis Before, During,and After a 7-Year Program. Infect Control Hosp Epidemiol 33: 338 -345. [Crossref]

12. Lanbeck P, Ragnarson Tennvall GFR (2016) A cost analysis of introducing an infectious disease specialist-guided antimicrobial stewardship in an area with relatively low prevalence of antimicrobial resistance. BMC Health Services Research 16: 311.

13. Akpan MR, Ahmad R, Shebl NA, Ashiru-Oredope D (2016) A Review of Quality Measures for Assessing the Impact of Antimicrobial Stewardship Programs in Hospitals. Antibiotics (Basel) 5. [Crossref]

14. Arif I, Assad S, Ghani U, Assad S (2016) Antibiotic Stewardship Program: A Dilemma for Control of Infections. Transl Surg 1: 79-82.

15. Nicolle LE (2014) Antimicrobial stewardship in long term care facilities: what is effective? Antimicrob Resist Infect Control 3: 6. [Crossref]

16. Bohannon J (2016) Models of effective antimicrobial stewardship programs.

17. Ministry of Health Malaysia (2014) Protocol on Antimicrobial stewardship program in healthcare facilities In Pharmaceutical Services Division, Medical Development Division and Family Health Development Division, Ministry of Health Malaysia.

18. Storey DF, Pate PG, Nguyen AT, Chang F (2012) Implementation of an antimicrobial stewardship program on the medical-surgical service of a 100-bed community hospital. Antimicrob Resist Infect Control 1: 32. [Crossref]

19. Aryee A, Price N (2015) Antimicrobial stewardship - can we afford to do without it? Br J Clin Pharmacol 79: 173-181. [Crossref]

20. Frieden TR, Bell B (2014) Core Elements of Hospital Antibiotic Stewardship Programs

21. Grunwald J, Zervos J, Zervos M, Brar I (2014) Antimicrobial stewardship: Strategies for a global response. CHRISMED J of Heal Res 1: 4-10.

Copyright: (C2017 Son NT. This is an open-access article distributed under the terms of the Creative Commons Attribution License, which permits unrestricted use, distribution, and reproduction in any medium, provided the original author and source are credited. 